\section{Grapefruit-medication interactions}

We are very concerned that statements made in a review article that appeared in $C M A J^{1}$ do not correctly reflect the current state of medical and scientific understanding of the topic of grapefruit-medication interactions.

We know of no validated evidence that coadministration of grapefruit juice with a drug has caused a dangerous interaction, resulting in serious adverse effects or actual harm to a patient's health. We point readers to 2 extensive review articles on grapefruit juice-drug interactions that have appeared in peerreviewed medical literature. ${ }^{2,3}$ These articles provide a review of primary research literature, a compilation of the extent of interactions with specific drugs, and an evaluation of their clinical importance; however, neither of these publications is cited in the CMAJ article.

We emphasize that Table 1 of the $C M A J$ review $^{1}$ shows theoretically predicted risks of grapefruit juice-drug interactions, as opposed to actual documented interactions. We are concerned that this generalized information may lead CMAJ readers to conclude incorrectly that grapefruit juice coadministration should be avoided for many more drugs than is supported by the scientific data. As a specific example, the interaction of grapefruit juice with atorvastatin is listed as high risk, with a dose-related adverse event of rhabdomyolysis. The authors go on to cite elsewhere in the article a published clinical study of addition of grapefruit juice co-treatment to patients taking atorvastatin for hyperlipidemia. ${ }^{4}$ The study results show a trivially small interaction of grapefruit juice with atorvastatin, with no evidence of adverse effects, let alone rhabdomyolysis. Other examples of incorrect information in Table 1 of the review include the purported interactions of grapefruit juice with quinine, quinidine, erythromycin, dextromethorphan, oxycodone, tolvaptan, nilotinib, and sunitinib, all of which are negligible and/or clinically unimportant in magnitude.
Table 2 of the CMAJ review presents case reports of adverse events supposedly related to grapefruit juicedrug interactions. In connection with the MedWatch system for spontaneous reporting of medication-related adverse events, the United States Food and Drug Administration is explicit in emphasizing the limitations of such reports. ${ }^{5,6}$ Unvalidated anecdotal reports do not establish cause and effect, and cannot be used to determine absolute or relative incidences of specific events. Adverse event case reports are to be used as "signals" to define whether further controlled scientific research is required to address a specific hypothesis. ${ }^{5-7}$ Looking again at the example of atorvastatin, the actual controlled study with chronic concomitant use of grapefruit juice and atorvastatin showed that there was no clinically significant interaction. ${ }^{4}$ In patients on extended stable atorvastatin treatment, addition of daily grapefruit juice in typical quantities slightly elevates serum atorvastatin concentrations, but has no meaningful effect on the serum lipid profile, and causes no detectable adverse liver or muscle effects. Modification of atorvastatin dosage when moderate amounts of grapefruit juice are co-ingested does not appear to be necessary.

We agree that grapefruit juice may produce clinically significant interactions with a few drugs. These include: simvastatin, buspirone, felodipine, and possibly cyclosporine. However, the number of affected drugs does not approach 85 . We encourage readers to visit www.DrugInteractionCenter.org, which provides a listing of drugs that are known to interact with grapefruit juice, the magnitude and clinical importance of the interaction, and citation of primary scientific documentation.

\section{David J. Greenblatt MD, Hartmut Derendorf PhD}

Professor of Pharmacology and Experimental Therapeutics (Greenblatt), Tufts University School of Medicine, Boston, Mass.; and Distinguished Professor and Chair, Department of Pharmaceutics (Derendorf), University of Florida, College of Pharmacy, Gainesville, Fla.
Competing interests: David Greenblatt and Hartmut Derendorf are clinical research advisors to the Florida Department of Citrus, Bartow, Fla.

\section{References}

1. Bailey DG, Dresser G, Arnold JMO. Grapefruitmedication interactions: Forbidden fruit or avoidable consequences? CMAJ 2013;185-309-16.

2. Mertens-Talcott SU, Zadezensky I, De Castro WV, et al. Grapefruit-drug interactions: can interactions with drugs be avoided? J Clin Pharmacol 2006;46: 1390-416.

3. Hanley MJ, Cancalon P, Widmer WW, et al. The effect of grapefruit juice on drug disposition. Expert Opin Drug Metab Toxicol 2011;7:267-86.

4. Reddy P, Ellington D, Zhu Y, et al. Serum concentrations and clinical effects of atorvastatin in patients taking grapefruit juice daily. $\mathrm{Br} \mathrm{J}$ Clin Pharmacol 2011;72:434-41.

5. Goldman SA. Limitations and strengths of spontaneous reports data. Clin Ther 1998;20 (Suppl C): C40-4

6. Ahmad SR. Adverse drug event monitoring at the Food and Drug Administration. J Gen Intern Med 2003; 18:57-60.

7. Venning GR. Validity of anecdotal reports of suspected adverse drug reactions: the problem of false alarms. Br Med J (Clin Res Ed) 1982;284:249-52.

CMAJ 2013. DOI:10.1503/cmaj.113-2109

\section{The authors respond}

Greenblatt and Derendorf ${ }^{1}$ have previously acknowledged their conflict of interest as clinical research advisors to the Florida Department of Citrus. That there is "no validated evidence" of "serious adverse effects" of an interaction of grapefruit with any affected medications is their opinion. We have documented published reports in the medical literature that are noted in our recent article. ${ }^{2}$

That a serious adverse event has not been reported should not be interpreted to mean that it has not happened. To recognize an adverse event, there first must be an awareness of the possibility of such an adverse event. Otherwise, the likelihood that it will be investigated is extremely low. This is mentioned in our article ${ }^{2}$ and is one of the major reasons why we were compelled to identify and inform the profession and public about this interaction.

Our article ${ }^{2}$ focuses on the number of new drugs now on the market that have the potential for serious toxicity if administered with grapefruit. The relevance of this aspect is highlighted by a recent publication showing that nearly one-quarter of the new drugs approved in Canada will eventually get a serious safety warning or have to be pulled 
from the market for safety reasons. Moreover, this ratio has increased to one-third for drugs given a priority review. Because we do have specific evidence for interactions between some medications and grapefruit, the public should be updated about new drugs where this interaction may be missed.

Is it sufficient that we identify these warnings and withdrawals only after they may have caused human suffering? After 20 years and hundreds of research publications on the topic of grapefruitdrug interaction, is there not enough well-documented science to predict with high likelihood the adverse effects and toxicity before unnecessary exposure? Our considered contention is that this is indeed the case. Moreover, a recent editorial in $B M J$ has lent further credence to the relevance of our conclusions. ${ }^{4}$

Even if the incidence of serious toxicity from a grapefruit-drug interaction was low in the patient population, which is as yet not fully known, the consequences would be dire (yet easily prevented). Moreover, why would you knowingly or even theoretically put yourself or others in harm's way? Caution is by far the wisest approach.

\section{David G. Bailey PhD}

Scientist, Lawson Health Research

Institute, London, Ont.

\section{References}

1. Greenblatt DJ, Derendorf H. Grapefruitmedication interactions [letter]. CMAJ 2013;185: 507.

2. Bailey DG, Dresser G, Arnold JMO. Grapefruitmedication interactions: Forbidden fruit or avoidable consequences? CMAJ 2013;185-309-16.

3. Lexchin J. New drugs and safety: what happened to new active substances approved in canada between 1995 and 2010? Arch Intern Med 2012; Oct. 8:1-2.

4. Pirmohamed M. Drug-grapefruit juice interactions. BMJ 2013;346:f1.

CMAJ 2013. DOI:10.1503/cmaj.113-2110

\section{Pregnancy and isotretinoin therapy}

We read with interest the article by Choi and colleagues on isotretinoin therapy and the importance of a multilevel approach to ensure adequate contraception in women taking potentially teratogenic medications. ${ }^{1}$ We agree that it is essential that health care providers know the failure rates of various contraceptive methods when counselling patients. However, we encourage health care providers to become familiar with the more recent and accepted perfect and typical use failure rates reported by Trussell and colleagues in Appendix 1, available at www.cmaj.ca /lookup/suppl/doi:10.1503/cmaj.1132111 /-/DC1, ${ }^{2}$ which in some instances are quite different than the ones the cited by Choi and colleagues. ${ }^{3}$

The rates reported by Trussell and colleagues are referred to in numerous contraception guidelines including those from the Society of Obstetricians and Gynaecologists of Canada, ${ }^{4}$ the Centers for Disease Control and Prevention, ${ }^{5}$ and the World Health Organization. ${ }^{6}$

Prescribers must also understand the difference between perfect-use and typical-use failure rates. Failure rates are reported as the percentage of women who will have an unintended pregnancy during the first year of use of a method. Perfect use may be hard to achieve, particularly with more compliance-demanding methods, which explains why typical-use failure rates are much higher than perfect -use failure rates with methods such as condoms and oral contraceptives. Longacting reversible contraceptive methods such as intrauterine contraceptive devices and implants are not as reliant on user compliance and hence typical-use failure rates approach those of perfect-use rates. Long-acting reversible contraceptives also have lower discontinuation rates at one year. ${ }^{2}$

We encourage contraceptive prescribers to be familiar with Trussell's reported failure rates, ${ }^{2}$ and reiterate that women of reproductive age who use teratogenic medications should be counselled about all contraceptive options - particularly long-acting reversible contraceptives given their low typical- and perfect-use failure rates and their increased adherence.

\section{Tania Dumont MD, Amanda Black MD} MPH

Assistant Professor (Dumont), Associate Professor (Black), The University of Ottawa; Pediatric and Adolescent Gynecologist (Dumont, Black), Children's Hospital of Eastern Ontario; obstetrician and gynecologist, the Ottawa Hospital (Dumont, Black); and Past Chair (Black), National Contraception Awareness Program, Ottawa, Ont.

\section{References}

1. Choi JS, Koren G, Nulman I. Pregnancy and isotretinoin therapy. CMAJ 2013;185:411-13.

2. Trussell J. Contraceptive efficacy. In: Hatcher RA, Trussell J, Nelson AL, et al. Contraceptive technology: twentieth revised edition. New York (NY): Ardent Media; 2011

3. General Practice Notebook. Warwickshire (UK): Oxbridge Solutions; 2013. Available: www .gpnotebook.co.uk/homepage.cfm (accessed 2013 Jan. 31).

4. Black A, Francoeur D, Rowe T. SOGC Clinical Practice Guidelines. Canadian Contraception consensus. J Obstet Gynaecol Can 2004;26:143-56.

5. US medical eligibility criteria for contraceptive use, 2010. MMWR Morb Mortal Wkly Rep 2010;59:5.

6. World Health Organization. Medical eligibility criteria for contraceptive use. 4th ed. Geneva (Switzerland): The Organization; 2010.

CMAJ 2013. DOI:10.1503/cmaj.113-2111

\section{The authors respond}

We thank Dumont and Black ${ }^{1}$ for further raising the issue of the need for effective contraception in order to prevent pregnancies during isotretinoin treatment.

Our report ${ }^{2}$ included only the typicaluse contraception failure rates. Please note that Box 1 in our manuscript provides the same typical-use failure rates as reported by Trussell and colleagues and quoted by Dumont and Black. ${ }^{2}$

June Seek Choi MD, Gideon Koren MD, Irena Nulman MD

The Motherisk Program (Choi, Koren, Nulman) Division of Clinical Pharmacology/Toxicology, Hospital for Sick Children, Toronto, Ont., and Cheil General, Hospital and Women's Healthcare Centre (Choi), Seoul, Republic of Korea.

\section{References}

1. Dumont T, Black A. Pregnancy and isotretinoin therapy [letter]. CMAJ 2013;185:508.

2. Choi JS, Koren G, Nulman I. Pregnancy and isotretinoin therapy. CMAJ 2013;185:411-13.

CMAJ 2013. DOI:10.1503/cmaj.113-2112

\section{Letters to the editor}

In submitting a letter, you automatically consent to have it appear online and/or in print. All letters accepted for print will be edited by $C M A J$ for space and style. Most references and multiple authors' names, full affiliations and competing interests will appear online only. (The full version of any letter accepted for print will be posted at cmaj.ca.) 